\title{
Two chemical-controlled switchable Cas9s for tunable gene editing
}

\author{
Meng Liang ${ }^{1,2}$, Yang Cui ${ }^{1,2}$, Jie Lan $^{1,2}$, Guangtao Song ${ }^{1,2}$, Jizhong Lou ${ }^{1,2 \bowtie}$ \\ ${ }^{1}$ Key Laboratory of RNA Biology, CAS Center for Excellence in Biomacromolecules, Institute of Biophysics, Chinese \\ Academy of Sciences, Beijing 100101, China \\ ${ }^{2}$ University of Chinese Academy of Sciences, Beijing 100049, China
}

Received: 21 January 2019 / Accepted: 24 February 2019 / Published online: 4 July 2019

\section{INTRODUCTION}

The clustered regularly interspaced short palindromic repeats (CRISPR) loci and their associated (Cas) genes are found in many bacterial and archaeal genomes as adaptive defense systems against phage infection and plasmid transfer (Mohanraju et al. 2016). In the CRISPRCas system, single or multiple Cas proteins were complexed with a small CRISPR RNA (crRNA), forming an RNA protein complex (RNP), which uses the crRNA as the guide to destruct the invading nucleic acids (Mohanraju et al. 2016). Based on CRISPR locus organization and the Cas gene content, CRISPR-Cas systems are divided into two classes. Class 1 systems use a complex of several Cas proteins called Cascade, while class 2 systems use a single Cas protein (such as Cas9), as interference module to recognize and cleave the target nucleic acids (Mohanraju et al. 2016). In 2013, a class 2 CRISPR-Cas system, CRISPR-Cas9, from Streptococcus pyogenes, was repurposed as a revolutionary tool for precise gene editing and gene expression control (Cong et al. 2013). In this system, the Streptococcus pyogenes Cas9 (SpyCas9) first binds to an artificial chimeric single-guide RNA (sgRNA) comprising crRNA and a transactivating crRNA (tracrRNA) module to form an effector complex (Cas9-sgRNA complex) (Jinek et al. 2012). After the recognition of a short protospaceradjacent motif (PAM) sequence in target DNA, Cas9sgRNA complex binds and induces an R-loop in target

Electronic supplementary material The online version of this article (https://doi.org/10.1007/s41048-019-0093-3) contains supplementary material, which is available to authorized users.

$\bowtie$ Correspondence: gsong@moon.ibp.ac.cn (G. Song), jlou@ibp.ac.cn (J. Lou)
DNA through base-pairing between the guide sequence in crRNA and the DNA target. SpyCas9 finally uses its $\mathrm{HNH}$ and RuvC nuclease domains, to cleave the target and nontarget strands, respectively (Fig. S1) (Jiang et al. 2016).

Despite much progress in CRISPR-Cas9 system in genome editing, numerous studies have assessed offtarget DNA binding and cleavage by the Cas 9 complex, both in vitro and in vivo (Fu et al. 2013; Hsu et al. 2013). Efforts to improve the Cas9 specificity have accordingly been of great interest in the field (Tycko et al. 2016). Two different strategies have been developed: engineering the high-fidelity Cas9 protein or guide RNA (Fu et al. 2014; Kleinstiver et al. 2016), spatiotemporal control of Cas9 or guide RNA via external trigger signals (Gao et al. 2016; Nihongaki et al. 2018; Richter et al. 2017). For the latter strategy, the Cas9 activity is controlled at the post-translational stage either by modifying Cas9 protein or sgRNA using an external stimulus such as chemicals or light. This conditional control of Cas9 function has the potential to provide spatiotemporal gene manipulation in user-defined cell population at a specific time and improve the accuracy of Cas9mediated genome editing. To date, diverse chemicalinducible Cas9s have been developed, including controlling assembly of split Cas9, fusing Cas9 with chemical-inducible protein domains. Another approach for controlling Cas 9 activity is using the photo-switchable protein domains to construct the light-inducible Cas9s (Nihongaki et al. 2018). These inducible Cas 9 systems have significantly expanded the CRISPR toolkits. It is known that during the target binding and cleavage by Cas9-sgRNA complex, the HNH domain undergoes a big structural transition from an inactivated state, in which the catalysis is hampered, to an activated state prone for 
the cleavage of the target strand (Fig. 1A). DNA cleavage of Cas 9 is controlled by the conformational state of the HNH nuclease domain. Here, we designed two types of switchable Cas 9 based on controlling the conformational switch of HNH domain by redox state or zinc ion of the surrounding buffers.

\section{RESULTS AND DISCUSSION}

The intracellular oxidation-reduction reactions play central roles in cell metabolism and are integral components of cellular signaling and cell fate decisions. Changes in the redox equilibrium of cells are accompanied with transitions of many cell functions, such as proliferation, differentiation, immune responses, senescence, and death (Banaszynski et al. 2006; Beal 1995). The ability to respond and control the intracellular redox equilibrium is thus of great interest in biomedical research. Redox condition is one of the most effective local stimuli that can be exploited. Alterations in the redox equilibrium are precipitated by changing either the glutathione/glutathione-disulfide (GSH/GSSG) ratio and/or the reduced/oxidized thioredoxin ratio. By placing artificial disulfides onto fluorescent proteins, several redox-sensitive GFPs were developed to probe the redox states of the cell (Belousov et al. 2006). In these designs, pairs of cysteines were introduced in different positions of the GFP such that the two residues are capable of forming an intramolecular disulfide bridge, resulting in a significant decrease of fluorescence intensity. These redox-sensitive GFPs (roGFPs) have been widely used to probe the intracellular redox state. Inspired by these designs, it is possible to construct a redox-sensitive Cas9 by controlling the conformation change of its active domain. Previous structural studies revealed that the distance between S867 and N1054 is less than $0.5 \mathrm{~nm}$ in spyCas9-sgRNA complex, whereas more than $5 \mathrm{~nm}$ in R-loop complex (Jiang et al. 2016). We thus incorporate a disulfide bound between HNH and Ruvc domain of Cas9 (S867C-N1054C), constructing a disulfide-controlled Cas 9 which is sensitive to the redox state of its surroundings.

We next isolate and purified the Cas 9 proteins with single or dual sites mutations, S867C, N1054C, and S867C/N1054C. The mobility shift analysis of the purified Cas 9 and its mutants under nonreducing conditions revealed that wtCas9 protein and its single-site mutants consist of a major band around $150 \mathrm{kDa}$, whereas the dual mutant consists of a major band around $190 \mathrm{kDa}$. This indicates that the isolated Cas 9 formed an intra- or inter-molecular disulfide bond. Boiling of Cas 9 in an SDS-PAGE buffer containing $5 \mathrm{mmol} / \mathrm{L}$ DTT converted slower component to the faster component similar to that of wtCas9 (Fig. 1B). We then asked whether the Cas9 activity could respond to the redox state of the surrounding conditions. The in vitro plasmid cleavage assay was next used to probe the redox-dependent Cas9
A

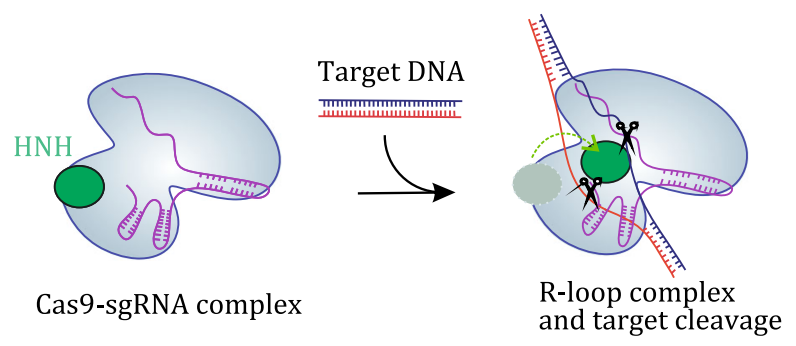

C

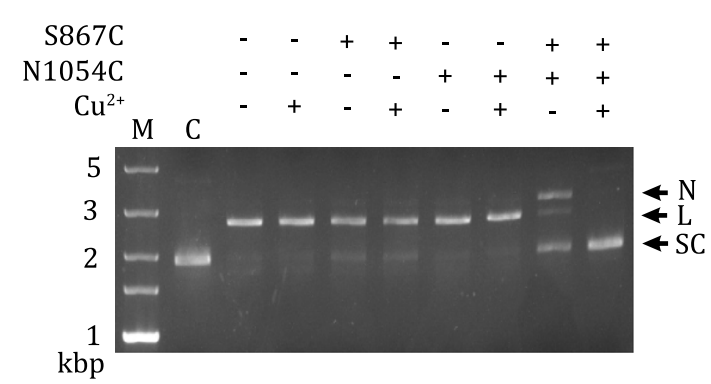

B

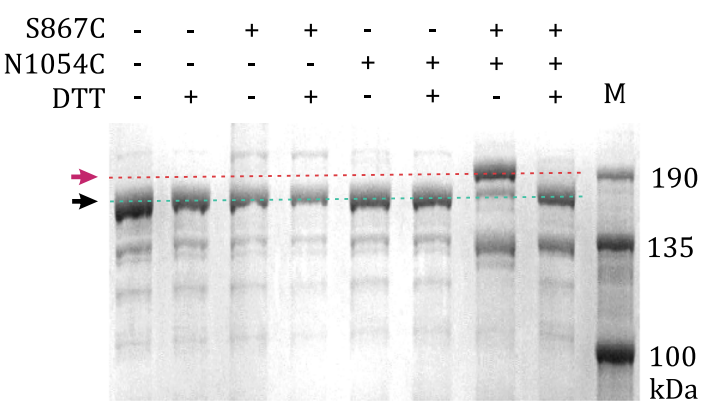

D

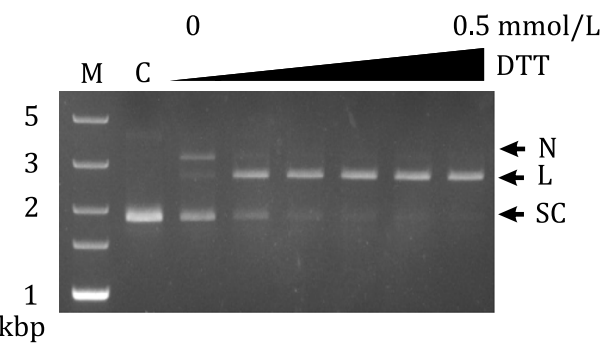

Fig. 1 A Schematic of the conformational change of SpyCas9 during R-loop formation. B SDS-PAGE analysis of the Cas 9 mutants. C Effect of $\mathrm{Cu}^{2+}$ on the target cleavage activity of Cas 9 and its mutants; D Effect of DTT on the target cleavage activity of Cas 9 and its mutants. SC: supercoiled DNA, L: linear DNA, N: nicked DNA 
activity. It is known that the $\mathrm{Cu}^{2+}$ could increase the degree of oxidization and induce disulfide bond. As shown in Fig. 2B, it could be found that the addition of $\mathrm{Cu}^{2+}$ decreases the redox-Cas9 activity, not the wtCas9, demonstrating that the target cleavage activity was switched off when increasing the oxidization state of the buffer. We next used DTT as the reducing agent to test the Cas9 activity. As shown in Fig. 1C, upon increase of the DTT concentration, the percentage of linearized plasmid increases. Generally, the intracellular redox changes are precipitated by changing either the GSH/ GSSG ratio and/or the reduced/oxidized thioredoxin ratio (Simonian and Coyle 1996). We thus used GSH/ GSSG ratio to probe the redox effect on Cas9 activity. It could be seen that after the increase of GSH/GSSG ratio, the target DNA could be totally linearized by redox-Cas 9 (Fig. S2). All these data showed that we have successfully constructed a switchable Cas9 which could be responsible to the redox state of buffer in vitro.

We also used a bacterial-based negative selection system to prove the function of the redox-Cas9 in vivo. As shown in Fig. 2A, a pET41a plasmid encodes a redoxCas 9 and sgRNA was first transformed into the E. coli Rosetta (DE3) strain. Another pUC19-based plasmid containing the target sequence of the sgRNA was then transformed to the Cas9-expressed E. Coli cells. The inactive Cas 9 cells which could not target and digest the plasmids enable cell survival due to the presence of an antibiotic-resistance gene, whereas cells with active Cas9 are depleted from the library. As expected, the dual-mutated redox Cas 9 showed much less activity than that of wtCas9 or single-site mutants (Fig. 2B). These data indicate that the redox state in E. Coli cell is moderate, which enable a high percent of disulfide bond. Unfortunately, we were not able to modulate the redox-Cas 9 activity by simply changing the concentration of DTT or ratio of GSH/GSSG (data not shown). Further work will focus on maximizing the design to screening new mutant which could effectively respond to the redox state in vivo.

Our previous biochemical studies revealed that the conformational switch of HNH domain in Cas9 during the R-loop formation could be altered by the single-site mutation in the linkers between HNH and RuvC domain (Zeng et al. 2018). We anticipated that the rotation of HNH domain could be also affected by changing the flexibility of the two linkers. To prove this hypothesis, we incorporate a zinc finger domain Zif268 (ElrodErickson et al. 1996) into the C-termini of the $\mathrm{HNH}$ between 908 and 909 in Cas9, constructing a zfCas9 (Fig. 2C). If there are no zinc ions in the solution, the zinc finger domain is unstable, and the conformation change of $\mathrm{HNH}$ will not be affected. When adding zinc ions into the solution, the domain will be stable and rigid, restraining the rotation of $\mathrm{HNH}$ and leading to a disable Cas9 activity. Figure 2D shows the target
A
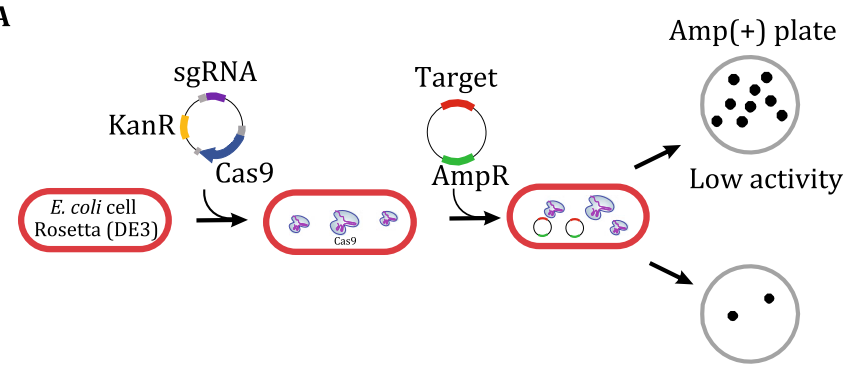

High activity
B

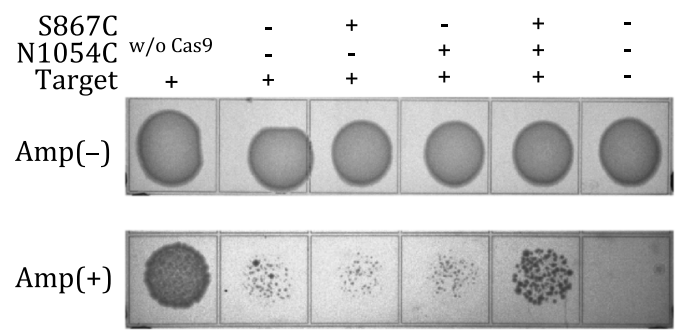

C

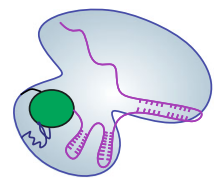

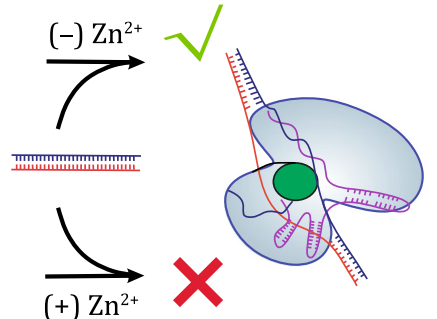

D

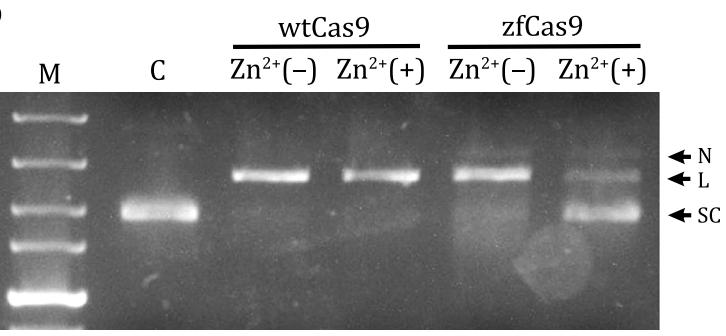

Fig. 2 A Schematic of bacterial-based target cleavage assay for the characterization of the disulfide bond-controlled switchable Cas9. B Transformation efficiencies of plasmids carrying target sequence to E. Coli cells expressing sgRNA and Cas 9 proteins. C Schematic of the conformational change of zfCas9 in the absence and presence of zinc ions. D Effect of zinc ion on the target cleavage activity of wtCas 9 and zfCas9. SC: supercoiled DNA, L: linear DNA, N: nicked DNA 
cleavage activity of the wtCas 9 and zfCas 9 in the absence and presence of zinc chloride $(0.2 \mathrm{mmol} / \mathrm{L})$. It could be found that the zfCas 9 could keep the target cleavage activity in the absence of zinc chloride similar to that of wtCas9. In contrast, after addition of $0.2 \mathrm{mmol} / \mathrm{L}$ zinc chloride into the reaction solution, the zfCas9 loses most of its cleavage activity. We next studied the $\mathrm{Zn}^{2+}$ concentration dependent on zfCas9 activity. We found that, upon increase of the $\mathrm{Zn}^{2+}$ concentration, the percentage of linearized plasmid decreases (Fig. S3). These results clearly demonstrate that the activity of zfCas 9 could be modulated by the zinc ions in the solution.

In summary, we have developed two switchable Cas9s for conditional control of gene editing. Through rational incorporation of a disulfide bond between its $\mathrm{HNH}$ and RuvC domain or a zinc finger domain in the linker between HNH and RuvC domain, we were able to modulate its target cleavage activity through changing the surrounding redox state or zinc ion concentration. As the redox equilibrium or zinc ion is an integral component of cellular signal processing and cell fate decision making, this redox-sensing gene editing tool may be very useful for the study of the molecular details of redox biochemistry.

\section{MATERIALS AND METHODS}

\section{Recombinant SpyCas9 expression and purification}

SpyCas9 and mutants were cloned into a custom pET41a expression vector which contains an $\mathrm{N}$-terminal GST-tag, His6-tag, and a thrombin protease cleavage site. Site-directed mutagenesis was performed to introduce point mutations into SpyCas9 and the accuracy was verified by DNA sequencing. Cas 9 and its mutants were expressed in E. coli Rosetta 2 (DE3) (Novagen) and purified by chromatography on Ni-NTA superflow (QIAGEN). The tags were removed by thrombin at $4{ }^{\circ} \mathrm{C}$ overnight, and purified by cation exchange (SP Sepharose) chromatography. The purified Cas9 proteins were then analyzed with SDS-PAGE gel electrophoresis.

\section{In vitro transcription and purification of RNA}

sgRNAs were generated by in vitro transcription from DNA templates carrying a T7-promoter sequence using T7 in vitro transcription kit (MEGAscript T7, Invitrogen) and purified by gel as described before (Zeng et al. 2018).

\section{Plasmid DNA cleavage assays}

pUC19-based protospacer plasmids were generated by annealed oligonucleotides between digested EcoR I and Hind III sites in pUC19 for further in vitro cleavage assays. Ligated plasmids DNA were transformed into E. coli DH5-alpha cells according to a standard heat shock protocol. A custom-designed plasmid containing a 20-bp DNA target sequence and a $5^{\prime}$-TGG-3' $3^{\prime}$ PAM motif was used in plasmid cleavage assays. Purified SpyCas9 and synthesized sgRNA were pre-incubated for $15 \mathrm{~min}$ in cleavage buffer $(20 \mathrm{mmol} / \mathrm{L}$ HEPES, pH 7.5 , $100 \mathrm{mmol} / \mathrm{L} \mathrm{KCl}, 2 \mathrm{mmol} / \mathrm{L} \mathrm{MgCl}_{2}$ ) to reach the final concentration of 125 and $375 \mathrm{nmol} / \mathrm{L}$, respectively; $500 \mathrm{ng}$ supercoiled plasmid was then added to the reaction mixtures and incubated at room temperature for various time points. The cleavage reactions were stopped by adding $6 \times$ sodium dodecyl sulfate loading buffer. $1.2 \%$ agarose gels were performed to characterize cleavage products. The gels were stained by Midori Green (NIPPON Genetics Europe) and analyzed with Image J software.

\section{Bacterial-based target cleavage assay}

PET41a-based plasmid containing wtCas9 or its mutants and sgRNA expression elements were first transformed into E. coli Rosetta (DE3) cells. These cells were further transformed with negative selection plasmids harboring cleavable target sites. Following a 60-min recovery in LB media, transformations were plated on LB plates containing chloramphenicol, kanamycin, and ampicillin. Cleavage of the negative selection plasmid was estimated by calculating the colony forming units per mg of DNA transformed.

Acknowledgements This work was supported by the National Natural Science Foundation of China (31771015, 11672317).

\section{Compliance with Ethical Standards}

Conflict of interest Meng Liang, Yang Cui, Jie Lan, Guangtao Song, and Jizhong Lou declare that they have no conflict of interest.

Human and animal rights and informed consent This article does not contain any studies with human or animal subjects performed by any of the authors.

Open Access This article is distributed under the terms of the Creative Commons Attribution 4.0 International License (http:// creativecommons.org/licenses/by/4.0/), which permits unrestricted use, distribution, and reproduction in any medium, provided you give appropriate credit to the original author(s) and the source, provide a link to the Creative Commons license, and indicate if changes were made. 


\section{References}

Banaszynski LA, Chen L-C, Maynard-Smith LA, Ooi AGL, Wandless TJ (2006) A rapid, reversible, and tunable method to regulate protein function in living cells using synthetic small molecules. Cell 126:995-1004

Beal MF (1995) Aging, energy, and oxidative stress in neurodegenerative diseases. Ann Neurol 38:357-366

Belousov VV, Fradkov AF, Lukyanov KA, Staroverov DB, Shakhbazov KS, Terskikh AV, Lukyanov S (2006) Genetically encoded fluorescent indicator for intracellular hydrogen peroxide. Nat Methods 3:281-286

Cong L, Ran FA, Cox D, Lin S, Barretto R, Habib N, Hsu PD, Wu X, Jiang W, Marraffini LA, Zhang F (2013) Multiplex genome engineering using CRISPR/Cas systems. Science 339:819-823

ElrodErickson M, Rould MA, Nekludova L, Pabo CO (1996) Zif268 protein-DNA complex refined at 1.6 angstrom: a model system for understanding zinc finger-DNA interactions. Structure 4:1171-1180

Fu Y, Foden JA, Khayter C, Maeder ML, Reyon D, Joung JK, Sander JD (2013) High-frequency off-target mutagenesis induced by CRISPR-Cas nucleases in human cells. Nat Biotechnol 31:822-826

Fu YF, Sander JD, Reyon D, Cascio VM, Joung JK (2014) Improving CRISPR-Cas nuclease specificity using truncated guide RNAs. Nat Biotechnol 32:279-284

Gao Y, Xiong X, Wong S, Charles EJ, Lim WA, Qi LS (2016) Complex transcriptional modulation with orthogonal and inducible dCas9 regulators. Nat Methods 13:1043-1049
Hsu PD, Scott DA, Weinstein JA, Ran FA, Konermann S, Agarwala V, Li Y, Fine EJ, Wu X, Shalem O, Cradick TJ, Marraffini LA, Bao G, Zhang F (2013) DNA targeting specificity of RNA-guided Cas9 nucleases. Nat Biotechnol 31:827-832

Jiang F, Taylor DW, Chen JS, Kornfeld JE, Zhou K, Thompson AJ, Nogales E, Doudna JA (2016) Structures of a CRISPR-Cas9 R-loop complex primed for DNA cleavage. Science 351:867-871

Jinek M, Chylinski K, Fonfara I, Hauer M, Doudna JA, Charpentier E (2012) A programmable dual-RNA-guided DNA endonuclease in adaptive bacterial immunity. Science 337:816-821

Kleinstiver BP, Pattanayak V, Prew MS, Tsai SQ, Nguyen NT, Zheng ZL, Joung JK (2016) High-fidelity CRISPR-Cas9 nucleases with no detectable genome-wide off-target effects. Nature 529:490-495

Mohanraju P, Makarova KS, Zetsche B, Zhang F, Koonin EV, van der Oost J (2016) Diverse evolutionary roots and mechanistic variations of the CRISPR-Cas systems. Science 353:aad5147

Nihongaki Y, Otabe T, Sato M (2018) Emerging approaches for spatiotemporal control of targeted genome with inducible CRISPR-Cas9. Anal Chem 90:429-439

Richter F, Fonfara I, Gelfert R, Nack J, Charpentier E, Moglich A (2017) Switchable Cas9. Curr Opin Biotechnol 48:119-126

Simonian NA, Coyle JT (1996) Oxidative stress in neurodegenerative diseases. Annu Rev Pharmacol Toxicol 36:83-106

Tycko J, Myer VE, Hsu PD (2016) Methods for optimizing CRISPRCas9 genome editing specificity. Mol Cell 63:355-370

Zeng Y, Cui Y, Zhang Y, Zhang Y, Liang M, Chen H, Lan J, Song G, Lou J (2018) The initiation, propagation and dynamics of CRISPRSpyCas9 R-loop complex. Nucleic Acids Res 46:350-361 